\title{
Unambiguous Detections of The Plane Symmetries That Noisy Experimental Images from Naturally Formed 2D Periodic Arrays of Physical Objects Most Likely Possess
}

\author{
Peter Moeck ${ }^{1}$
}

${ }^{1}$ Department of Physics, Portland State University, Portland, OR, USA

Computer based Crystallographic Image Processing (CIP) of naturally formed arrays of physical objects that are periodic in two-dimensions (2D) originated with the electron crystallography community some 40 years ago [1] and was recently applied to noisy images that were recorded from regular 2D periodic arrays of molecules and atoms with scanning tunneling microscopes (STMs) [2,3]. The procedures of the latter studies were analyzed theoretically [4,5] and can be generalized to all kinds of scanning probe microscopes (SPMs). CIP for SPM removes the effects of multiple-mini tips on a blunt scanning probe tip and suppresses random noise effectively [3-5]. The former of these two applications of CIP is beyond the original inception of the technique as applied to high-resolution transmission electron microscope images that were recorded from crystals either with parallel illumination [1] or in the scanning probe [6] mode.

An unresolved issue in CIP is the somewhat arbitrary choice of which plane symmetry group (2D space group) to enforce on the data that are to be extracted from noisy experimental images. While electron crystallographers typically employ their chemical intuition and often possess projections from multiple 3D directions into the two dimensions of their images, see discussions in appendix A of ref. [4], the application of CIP to SPM images would become much more popular if there were an objective and statistically sound procedure for the detection of the plane symmetry groups that noisy experimental images from regular 2D periodic arrays of atoms or molecules most likely possess. Note that it is either the prior knowledge (or an educated guess) of the correct symmetry group or its unambiguous identification that enable both $(i)$ the crystallographic averaging over the $2 \mathrm{D}$ periodic motif in order to extract more detailed information on the physical entities in the array and (ii) the most effective suppression of random noise in the image.

Because there are symmetry group inclusion relations to be considered, model selections by a geometric Akaike information criterion (G-AIC) [7] seems to be an obvious choice for such an objective procedure. A G-AIC has been utilized for the detection of frieze symmetries in 1D periodic patterns and it was suggested that this approach could be generalized to 2D periodic patterns [8]. The model selections was in ref. [8] done in direct space as only a few unit cell repeats were available. The motif (and unit cell) selections in ref. [8] did not follow the well known crystallographic restrictions that are due to the spatial distribution of site symmetries in the seventeen 2D space groups in their standard settings [9]. No distinction was made between images from naturally occurring and artificially* created 2D periodic patterns throughout ref. [8]. Instead of generalizing the ideas in [8] to 2D, we utilize for our new G-AIC procedure a traditional Fourier transform and Bart Verberck's recently derived symmetry-adapted Fourier back transforms [10].

In our new procedure (that is to be laid out in detail elsewhere), the complex-valued Fourier coefficients of an image of a 2D periodic array are extracted by a traditional Fourier transform. From these coefficients, Verberck's symmetry-adapted Fourier back transforms [10] are calculated for each of the 17 plane symmetry groups. Sums of squared difference measures, i.e. Kenichi Kanatani's residuals $J$ [7], between the pixelwise intensity in the experimental image, $I_{\text {exp-x,y,n}}$, and the corresponding normalized intensities of the symmetryadapted back transforms, ${ }^{1} / M F_{\text {Verberck-x,y,n}}$, are then calculated, where $M$ stands for the multiplicities of the general position [9] per lattice point and $x, y$ are pixel labels that are related to integer fractions of the $n$ 2D unit cell(s). Higher symmetric plane symmetry groups, designated by primes, within all of the applicable branches of the hierarchy of Figure 1 are then to be selected as long as the inequalities $J^{\prime}<f_{i} \cdot J$ hold for the sums of squared difference measures of the higher (primed) and lower (unprimed) symmetric plane groups. The factors $f_{i}(>1)$ are specific to the transitions between nodes in the plane symmetry group hierarchy, Fig. 
1, and include relations between the multiplicities of the general/special positions [9] of the 2D space groups.

Note that we utilized earlier a G-AIC for the unambiguous detection of the 2D Bravais lattices that underlie noisy 2D periodic images [4]. Since multiple scanning probe mini-tips cannot affect the translation symmetry that an image of a regular 2D periodic array acquires in the sequential recording process, the application of that particular G-AIC reveals blunt tip effects on SPM images. These effects may then, as already mentioned above, be corrected by CIP and random noise in the scanning apparatus be suppressed in the process [3-5].

For our crystallographic averaging and supporting G-AIC methods [2-5] to produce satisfactory results, one needs to make sure that $(i)$ at least some 50 repeats of the $2 \mathrm{D}$ unit cells are available for the analysis, that (ii) random errors with a Gaussian distribution dominate the data, and that (iii) a blunt scanning probe tip does not possess a point symmetry higher than unity. Finally, note that all of our methods are (just as all of the traditional CIP procedures) completely independent of the type of microscope with which the 2D periodic images have been recorded. It is straightforward to generalize our methods to quasicrystalline 2D arrays [11].

\section{References}

[1] A. Klug, Chemica Scripta 14 (1978-79) 245-256

[2] P. Moeck et al., Mater. Res. Soc. Symp. Proc. 1318 (2011) 149-154; P. Moeck et al., Proc. $11^{\text {th }}$ IEEE Intern. Conf. Nanotech. (2011) 891-896; doi:10.1109/NANO.2011.6144508, see: http://ieeexplore.ieee.org/ [3] P. Moeck, in: Microscopy: Science Technology, Applications and Education, no. 4, vol. 3 (2010), eds. A. Méndez-Vilas and J. Diaz (Formatex Microscopy Series), p. 1951-1962; open access:

http://www.formatex.info/microscopy4/1951-1962.pdf

[4] J. C. Straton et al., Cryst. Res. Technol. 49 (2014) 663-680

[5] J. C. Straton et al., J. Advanced Microsc. Res. 9 (2014) 206-216; J. Straton et al., Adv. Struct. Chem.

Imaging 1 (2015) 14; open access: http://ascimaging.springeropen.com/articles/10.1186/s40679-015-0014-6

[6] D. G. Morgan et al., J. Electron Microsc. 58 (2009) 223-244

[7] K. Kanatani, Intern. Journal of Computer Vision 26 (1998) 171-189

[8] Y. Liu et al., IEEE Trans. Pattern Analysis and Machine Intellig. 26 (2004) 354-371

[9] T. Hahn (ed.), Brief Teaching Edition of Volume A, Space-group symmetry, International Tables for Crystallography, $5^{\text {th }}$ revised edition, International Union of Crystallography, Chester (2005)

[10] B. Verberck, Symmetry 4 (2012) 379-426; open access: http://www.mdpi.com/2073-8994/4/3/379

[11] Funding from Portland State University's Faculty Enhancement program is gratefully acknowledged.

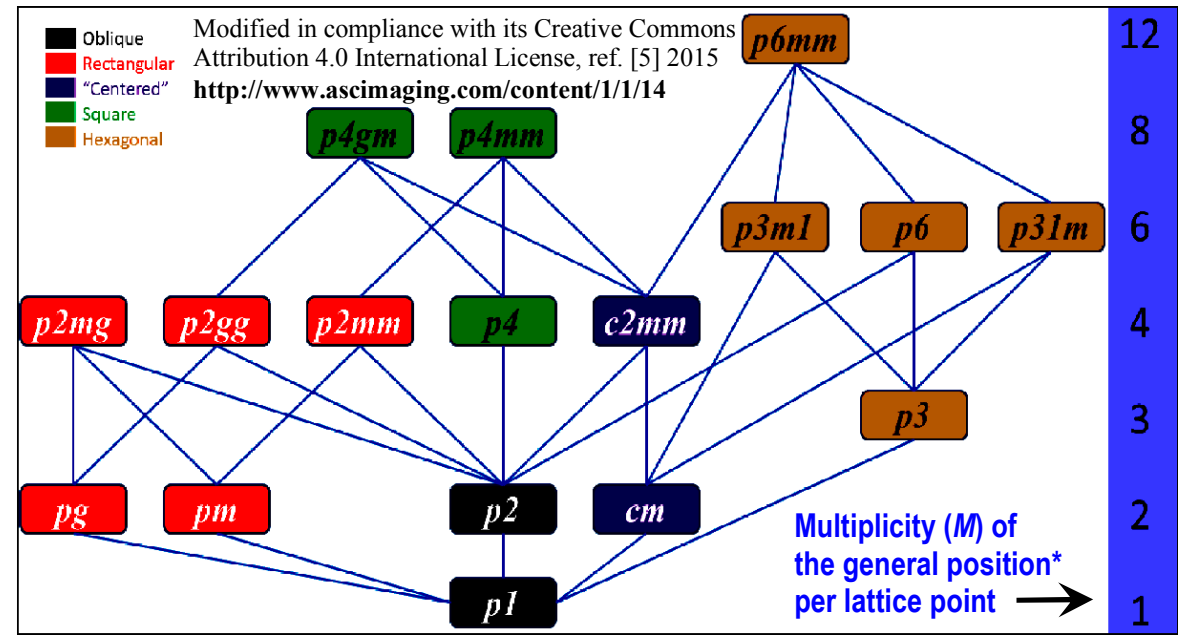

Figure 1. Hierarchy of the 17 plane symmetry groups (in Hermann-Mauguin notation [9]), color coded to aid the assignments of their corresponding 2D Bravais lattices. Tanslationengleiche (Type I, [9]) minimal supergroups and maximal subgroups are connected by straight lines. (Some of the nodes in this contracted graph refer to conjugate subgroups.)

* Artificially created 2D periodic patterns, (e.g. textiles, wallpapers, M. C. Escher's 2D periodic drawings, building decorations in the Arabic/Moorish/Islamic traditions, ...) may possess non-crystallographic site symmetries and plane color symmetries. They also do not need to obey the crystallographic symmetry restrictions that nature typically imposes on the equilibrium positions of atoms and molecules (in naturally formed) regular 2D periodic arrays. 